Research Paper

\title{
CFP is a prognostic biomarker and correlated with immune infiltrates in Gastric Cancer and Lung Cancer
}

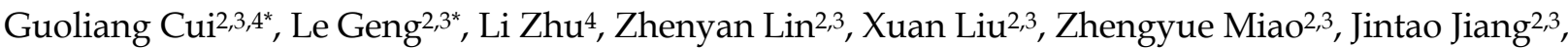 \\ Xiaoke Feng ${ }^{2,3}$ and Fei Wei ${ }^{\square}$ \\ 1. Department of Physiology, School of medicine \& Holistic Integrative Medicine, Nanjing University of Chinese Medicine, Nanjing 210023, Jiangsu, China. \\ 2. Department of Traditional Chinese Medicine, The First Affiliated Hospital of Nanjing Medical University, Nanjing 210029, Jiangsu, China. \\ 3. Institute of Integrated Chinese and Western Medicine, Nanjing Medical University, Nanjing 210029, Jiangsu, China. \\ 4. The Second Affiliated Hospital of Nanjing University of Chinese Medicine, Nanjing 210017, Jiangsu, China. \\ *These authors contributed equally to this work.
}

$\triangle$ Corresponding authors: Fei Wei, Department of Physiology, School of medicine \& Holistic Integrative Medicine, Nanjing University of Chinese Medicine, Nanjing 210023, Jiangsu, China. E-mail: weifei@njucm.edu.cn; Xiaoke Feng, Department of Traditional Chinese Medicine, The First Affiliated Hospital of Nanjing Medical University, Nanjing 210029, Jiangsu, China. Institute of Integrated Chinese and Western Medicine, Nanjing Medical University, Nanjing 210029, Jiangsu, China. E-mail: fengxiaoke@njmu.edu.cn.

(c) The author(s). This is an open access article distributed under the terms of the Creative Commons Attribution License (https://creativecommons.org/licenses/by/4.0/). See http://ivyspring.com/terms for full terms and conditions.

Received: 2020.07.19; Accepted: 2021.03.21; Published: 2021.04.12

\begin{abstract}
Complement factor properdin (CFP), encodes plasma glycoprotein, is a critical gene that regulates the complement pathway of the innate immune system. However, correlations of CFP in cancers remain unclear. In this study, the expression pattern and prognostic value of CFP in pan-cancer were analyzed via the Oncomine, PrognoScan, GEPIA and Kaplan-Meier plotters. In addition, we used immunohistochemical staining to validate CFP expression in clinical tissue samples. Finally, we evaluated the correlations between CFP and cancer immune infiltrates particularly in stomach adenocarcinoma (STAD) and lung adenocarcinoma (LUAD) by using GEPIA and TIMER databases. The results of database analysis and immunohistochemistry showed that the expression level of CFP in STAD and LUAD was lower than that in normal tissues. Low expression level of CFP was associated with poorer overall survival (OS), first progression (FP), post progression survival (PPS) and was detrimental to the prognosis of STAD and LUAD, specifically in stage 3, stage T3, stage N2 and N3 of STAD $(P<0.05)$. Moreover, expression of $C F P$ had significant positive correlations with the infiltration levels of CD8+ T cells, CD4+ T cells, macrophages, neutrophils and dendritic cells (DCs) in STAD and LUAD. Furthermore, gene markers of infiltrating immune cells exhibited different CFP-related immune infiltration patterns such as tumor-associated-macrophages (TAMs). These results suggest that CFP can serve as a prognostic biomarker for determining prognosis and immune infiltration in STAD and LUAD.
\end{abstract}

Key words: complement factor properdin; gastric cancer; lung cancer; tumor microenvironment; prognostic biomarker

\section{Introduction}

The latest statistics from China Cancer Center in 2019 showed that malignant tumors account for $23.91 \%$ of all deaths of the Chinese population as a result of malignant tumors continue on the rise at a cost exceeding 220 billion each year [1]. Gastric cancer, lung cancer, liver cancer and breast cancer remained the most common malignant tumors which pose a serious threat to human health and life. In particular, occurrence of lung cancer and gastric cancer separately ranked first and second among the male, and separately ranked first and third in number of deaths as a result of tumor $[2,3]$. These two kinds of tumors are highly heterogeneous, with poor effective treatments and poor prognosis, and the 5-year survival rate of lung cancer was as low as $15 \%$ [2]. Relevant studies [4,5] have shown that delayed diagnosis and extensive metastasis are the main reasons for the poor prognosis of gastric cancers and lung cancers. As traditional molecular targeted therapy demonstrated relatively slow progress, patients with advanced gastric cancer were just finitely benefited from the current chemotherapy combined with targeted therapy. Therefore, it is necessary to further identify the panoramic molecular 
characteristics of tumors and explore new biomarkers and molecular targets related to prognosis and efficacy judgment, so as to find more effective treatment strategies.

The occurrence and development of tumors are influenced by the complex interaction between a variety of immune cells in the tumor microenvironment (TME) and tumor cells [6, 7]. Besides, studies have showed that natural immune cells (macrophages, neutrophil, dendritic cells, lymphocyte, myeloid suppressor cells, and natural killer cells) and acquired immune cells ( $\mathrm{T}$ cells and B cells) in the TME have different variables, which participate in the process of promoting or inhibiting tumor growth respectively, and have certain value for the prognosis of cancers [6]. Whole-genome expression analysis has begun to provide important molecular information for tumor-induced lymphocyte infiltration and myeloid cell reorganization. However, the interaction between the tumor and its microenvironment has not been fully elaborated, neither is the mechanism related to immune infiltration in tumor microenvironment, both of which deserves further study.

$C F P$, encodes a plasma glycoprotein, binds and stabilizes the unstable $\mathrm{C} 3$ convertases $(\mathrm{C} 3 \mathrm{bBb})$ in the complement system, playing a positive role in regulating the natural immune system in alternative pathway (AP) [8]. CFP is mainly synthesized and/or secreted by white blood cells. In addition, a variety of immune cells have a significant effect on serum CFP expression level, especially mature neutrophils. Recent studies have shown that in cases of patients receiving chemotherapy, CFP level would be reduced by $19-32 \%$ as the neutrophil decreases [9]. Several other studies have revealed that CFP may be indirectly associated with tumor progression and invasion through complement cascade [10]. Complement inhibition as a potential concept for cancer therapy has been studied in recent years [11]. While latest studies [12] have shown that CFP up-regulates TES mediated transcription factor DDIT3 to inhibit the growth of breast cancer cells, the relationship between CFP and other tumors has not been reported. In particular, the relationship between tumor prognosis and tumor immune microenvironment remains unclear.

In this study, we used the TIMER database and immunohistochemical staining to evaluate the expression of CFP in tumors, and then used the PROGNSCAN, GEPIA and Kaplan-Meier database to study the relationship between CFP and the prognosis of different tumors. TIMER and GEPIA database were used to explore the potential relationship between CFP expression and tumor immune invasion. The results showed that CFP is closely related to the prognosis of STAD and LYAD, and potentially interact with tumor immune infiltration.

\section{Materials and methods}

\section{Oncomine Database Analysis}

The Oncomine database (https://www. oncomine.org) collects cancer-related chip information from various sources, including 715 data sets with a total of 86,733 sample information. We analyzed the expression of CFP in different tumors in this database. Then we ranked the tumors according to different expression levels and set the $P$ value as 0.001 , the fold change as 1.5.

\section{TIMER Database Analysis}

TIMER (https://cistrome.shinyapps.io/timer/) is a comprehensive resource database for detecting the infiltration of immune cells in tumor tissues using RNA-SEQ expression profile data. We first explored the Gene differential expression between tumor tissue and normal tissue using the Diff Exp module in the database, and then analyzed the expression of CFP in different cancers using the Gene module. The correlation module was used to further investigate the correlation between CFP expression and the gene markers of tumor infiltrating immune cells to reveal the correlation between CFP and the abundance of immune infiltrates (including B cells, CD4+ T cells, CD8+ $\mathrm{T}$ cells, neutrophils, macrophages, and dendritic cells). Gene markers were selected from the previous researches. The correlation module generated the expression scatter plots between a pair of users defined genes in a given cancer type, together with the Spearman's correlation and the estimated statistical significance. CFP was plotted on the x-axis, while marker genes were plotted on the y-axis. The gene expression level was displayed with log2 RSEM.

\section{PrognoScan Database and Kaplan-Meier Plotter Database Analysis}

We analyzed the correlation between CFP expression and survival in various types of cancers by the PrognoScan database (http://www.abren.net/ PrognoScan/). PrognoScan searches for relationships between gene expression and patient prognosis, such as overall survival (OS), disease-free survival (DFS) and so on, across a large collection of publicly available cancer microarray datasets. The threshold was adjusted to a Cox $P$-value $<0.05$.

The Kaplan-Meier plotter can assess the effect of 54,675 genes on survival using 10,461 cancer samples. The correlation between CFP expression and survival in gastric cancer, lung adenocarcinoma, lung squamous cell carcinoma, breast invasive carcinoma 
and ovarian cancer was analyzed by Kaplan-Meier plotter (http://kmplot.com/analysis/). This threshold was $\log$ rank $P$ value $<0.05$ in Kaplan-Meier plotter database.

\section{Gene Correlation Analysis in GEPIA}

The RNA-Seq datasets GEPIA used are based on the UCSC Xena project (http://xena.ucsc.edu), and are computed by a standard pipeline. This is also a cancer data mining site, mainly based on TGCA and GTEx Projects. Correlations between CFP and genes markers of B cells, macrophages, and monocytes were analyzed in GEPIA. Similar to the TIMER database, we analyzed the tumor and normal tissue data, and the correlation coefficients were determined by the Spearman method. CFP was plotted on the x-axis while marker genes were plotted on the y-axis.

\section{UALCAN Database Analysis}

UALCAN (http://ualcan.path.uab.edu/index. $\mathrm{html}$ ) provides protein expression analysis option using data from Clinical Proteomic Tumor Analysis Consortium (CPTAC) Confirmatory/Discovery dataset. We analyzed the protein expression of CFP in colon cancer, breast cancer, ovarian cancer, clear cell renal cell carcinoma and uterine corpus endometrial carcinoma. Z-values represent standard deviations from the median across samples for the given cancer type. Log2 spectral count ratio values from CPTAC were first normalized within each sample profile, then normalized across samples.

\section{Human tissue specimens and immuno- histochemistry}

Human normal lung tissue $(\mathrm{n}=7)$, lung adenocarcinoma tissue $(n=7)$, normal gastric tissue $(n=12)$, and stomach adenocarcinoma tissue $(n=12)$ were collected from patients at The Second Affiliated Hospital of Nanjing University of Chinese Medicine (Nanjing China). Informed consent was obtained from each patient and this study was approved by the Ethics Committee of The Second Affiliated Hospital of Nanjing University of Chinese Medicine (2020SEZ-027-02). The inclusion criteria for patients were as follows: 1) no history of neoadjuvant therapy, 2) underwent resection, 3) no history of malignant tumour other than LUAD or STAD, 4) postoperative pathology confirmed gastric or pulmonary adenocarcinoma. Exclusive criteria were: 1) human immunodeficiency virus-positive, evidence of acute infection, or concomitant autoimmune disease requiring immunosuppressive therapy at the time of surgery, 2) received chemotherapy, radiation, or any other treatment for the cancer before surgery, 3) incomplete clinical information. The clinical and pathological features of the cohort of patients are illustrated in Supplementary Table 2. The Paraffinembedded tissue sections were dewaxed in xylene and then immersed in gradient alcohol. The sections were incubated with $3 \% \mathrm{H}_{2} \mathrm{O}_{2}$ to remove endogenous peroxidase and then immersed in antigenic repair buffer for antigenic retrieval at high temperature and pressure. Primary antibody (rabbit polyclonal anti-CFP antibody, dilution: 1:50, Proteintech) was applied to the sections overnight at $4{ }^{\circ} \mathrm{C}$, and after washing, horseradish peroxidase-labeled anti-rabbit IgG was added as secondary antibody for $2 \mathrm{~h}$ at room temperature. Followed by DAB staining, hematoxylin nuclear staining, dehydration and mounting. The sections were observed at $200 \times$ magnification using an optical microscope and Image-Pro Plus 6.0 professional image analysis software was used to analyze and quantitate the area of positive expression and integrated optical density (IOD). Mean optical density (MOD) was used to express the relative expression levels of CFP. MOD $=\mathrm{IOD} /$ positive area.

\section{Statistical Analysis}

The results generated in Oncomine are presented with $P$-values determined in t-tests, fold changes, and gene ranks. The Kaplan-Meier method was used to estimate the survival curve. To compare the survival curves, we used the log rank test to calculate the HR and logrank $P$-value in Kaplan-Meier Plotter and GEPIA. The correlation of gene expression was evaluated using Spearman's correlation and statistical significance, and the strength was determined using the following guide for the absolute value: 0.00-0.19 "very weak", 0.20-0.39 "weak", 0.40-0.59 "moderate", 0.60-0.79 "strong", $0.80-1.0$ "very strong". Quantitative data was expressed as mean \pm standard deviation. Inter-group comparison of quantitative data that conformed to a normal distribution was carried out using the two-sample t-test of the means. $P$ $<0.05$ was considered statistically significant.

\section{Results}

\section{The mRNA expression levels of CFP in different type of human cancers}

In order to determine the expression levels of CFP in different tumor tissues, we analyzed the expression levels of CFP in the Oncomine database. The results revealed that the expression levels of CFP in kidney cancer, and lymphoma were higher compared with the normal tissues, while in bladder cancer, brain and CNS cancer, breast cancer, colorectal cancer, leukemia, liver cancer, lung cancer, myeloma, ovarian cancer and sarcoma decreased significantly (Figure 1A). 
A

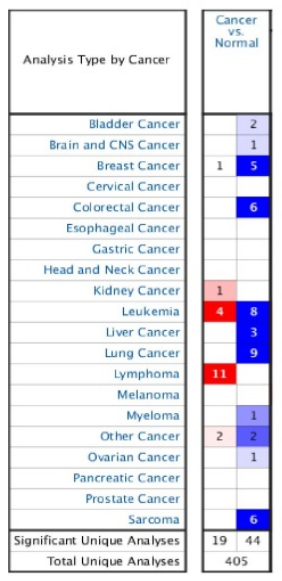

B

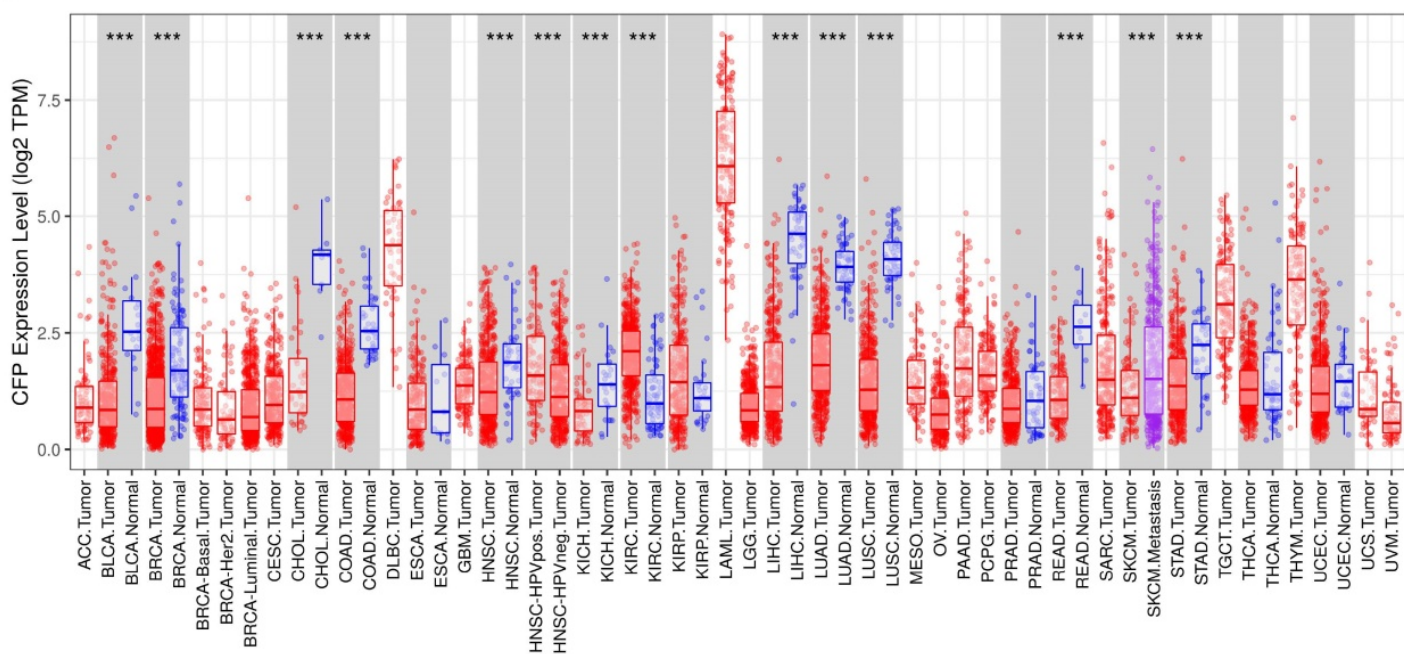

Figure 1. The expression levels of CFP in cancers. (A) Compared with normal tissues, the expression levels of CFP in different cancers via the Oncomine database. (B) Human CFP expression levels in different tumor types from TCGA database were determined by TIMER $(* P<0.05, * * P<0.01, * * * P<0.001)$

To further evaluate CFP expression in human cancers, we examined RNA-seq data of multiple malignancies in TCGA (Figure 1B). The expression levels were lower than adjacent normal tissues in BLCA (bladder urothelial carcinoma), BRCA (breast invasive carcinoma), CHOL (cholangiocarcinoma), COAD (colon adenocarcinoma), HNSC (head and neck cancer), $\mathrm{KICH}$ (kidney chromophobe), LHIC (liver hepatocellular carcinoma), LUAD (lung adenocarcinoma), LUSC (lung squamous cell carcinoma), READ (rectum adenocarcinoma) and STAD (stomach adenocarcinoma). Meanwhile, CFP expression was significantly higher in HNSC-HPV positive (head and neck squamous carcinoma-HPV positive) and KIRC (kidney renal clear cell carcinoma).

\section{The protein expression level of CFP in cancers}

Based on the UALCAN database, we found that the protein expression level of CFP in the primary tissue of breast cancer, colon cancer, lung adenocarcinoma, ovarian cancer and uterine corpus endometrial carcinoma were lower than in normal tissues, however, we did not obtain a significant difference in clear cell renal cell carcinoma (Figure 2A-F). Immunohistochemical staining was used to verify the expression level of CFP in LUAD and STAD, and the results indicated that the protein expression levels of CFP were significantly decreased in LUAD and STAD compared with the normal tissues (Figure 2G-H).

\section{Prognostic value of CFP in different cancers}

To determine whether CFP is a promoter or suppressor of tumors, we further analyzed the relationship between CFP expression and prognosis in these tumors. First, we used PrognoScan to explore the relationship between CFP expression and prognosis of different cancer. Notably, CFP expression significantly impacted prognosis in 8 cancer types, namely, blood cancer, brain cancer, breast cancer, lung cancer, ovarian cancer and soft tissue cancer (Figure 3). In blood cancer (OS: total number $=58, \mathrm{HR}=1.33$, Cox $P=0.014354)$, brain cancer (OS: total number $=74, \mathrm{HR}=3.52$, Cox $P=$ 0.002613 ) and breast cancer [RFS (relapse-free survival): total number $=60, \mathrm{HR}=1.64, \operatorname{Cox} P=$ 0.034006; DMFS (distant metastasis-free survival): total number $=286, \mathrm{HR}=0.69, \mathrm{Cox} P=0.040377], C F P$ played a detrimental role. In contrast, CFP played a protective role in lung cancer [OS: total number $=204$, $\mathrm{HR}=0.70$, Cox $P=0.032340 ;$ RFS (relapse-free survival): total number $=204, \mathrm{HR}=0.71$, Cox $P=$ 0.00543 ], ovarian cancer (OS: total number $=278, \mathrm{HR}$ $=0.45, \operatorname{Cox} P=0.031022)$ and soft tissue cancer (DMFS (distant metastasis-free survival): total number $=140$, $\mathrm{HR}=0.04, \operatorname{Cox} P=0.000000$ ).

Similarly, we used the Kaplan Meier plotter to further examine the relationship between CFP and prognosis in different cancers. Interestingly, the poor prognosis in gastric cancer $(\mathrm{OS} \mathrm{HR}=0.66,95 \% \mathrm{CI}=$ 0.54 to $0.8, P=3.1 \mathrm{e}-0.5$; FP HR $=0.72,95 \% \mathrm{CI}=0.57$ to $0.91, P=0.0058$; PPS HR=0.65, $95 \% \mathrm{CI}=0.5$ to $0.84, P=$ $0.0011)$, lung adenocarcinoma (OS HR $=0.6,95 \% \mathrm{CI}=$ 0.45 to $0.8, P=0.00046$; FP HR $=0.61,95 \% \mathrm{CI}=0.45$ to $0.85, P=0.0026$ ) and breast invasive carcinoma (OS $\mathrm{HR}=0.69,95 \% \mathrm{CI}=0.56$ to $0.86, P=0.00087$; PPS HR $=0.77,95 \% \mathrm{CI}=0.6$ to $0.98, P=0.033$; RFS HR $=0.74$, $95 \% \mathrm{CI}=0.66$ to $0.84, P=1.2 \mathrm{e}-06$; DMFS HR $=0.73$ $95 \% \mathrm{CI}=0.59$ to $0.89, P=0.0024)$ was shown to be correlated with lower CFP expression (Figures 4A-F, 
J-M). However, CFP expression exerted less influence on ovarian cancer besides PFS (Figures 4N-P), and showed a better relation with FP and PPS in lung squamous cell carcinoma (Figures $\mathbf{4 H}, \mathbf{I}$ ). These results suggested that the CFP expression had an impact on the prognosis of stomach adenocarcinoma, lung adenocarcinoma and breast invasive carcinoma.

\section{Low CFP expression impacts the stratified STAD population}

In order to explore the relationship between CFP and gastric cancer, and its possible mechanism in gastric cancer, we used the Kaplan-Meier plotter database to analyze the relationship between CFP expression and clinical characteristics of gastric cancer patients. Low expression of CFP was associated with worse survival in male and female patients as well as Lauren classification and differentiation $(P<0.05)$. Besides, in stage 3, stage T3, stage N2 and N3, low expression of CFP was associated with worse survival in gastric cancer $(P<0.05)$.
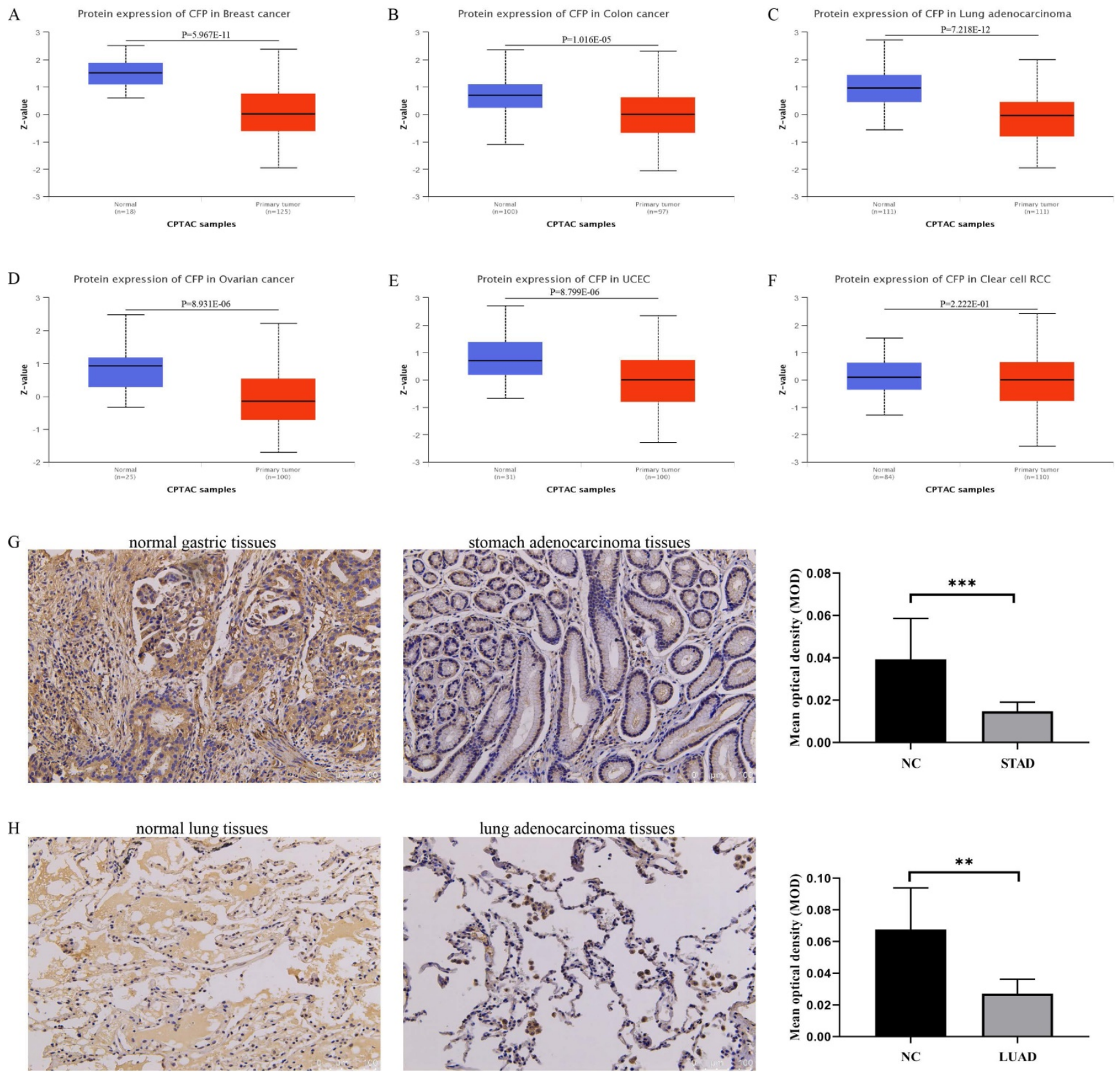

Figure 2. The protein expression levels of CFP in cancers. (A-F) Human protein expression of CFP in different tumor types from Clinical Proteomic Tumor Analysis Consortium (CPTAC) Confirmatory/Discovery dataset were determined by UALCAN. Clear cell renal cell carcinoma (RCC), Uterine corpus endometrial carcinoma (UCEC). Z-values represent standard deviations from the median across samples for the given cancer type. (G) Immunohistochemical results showed the expression levels of CFP in normal gastric tissues and stomach adenocarcinoma tissues. $(\mathrm{H})$ Immunohistochemical results showed the expression levels of CFP in normal lung tissues and lung adenocarcinoma tissues. Magnification, $\times 200 . * * P<0.01, * * * P<0.001$. NC, negative control; STAD, stomach adenocarcinoma; LUAD, lung adenocarcinoma. 

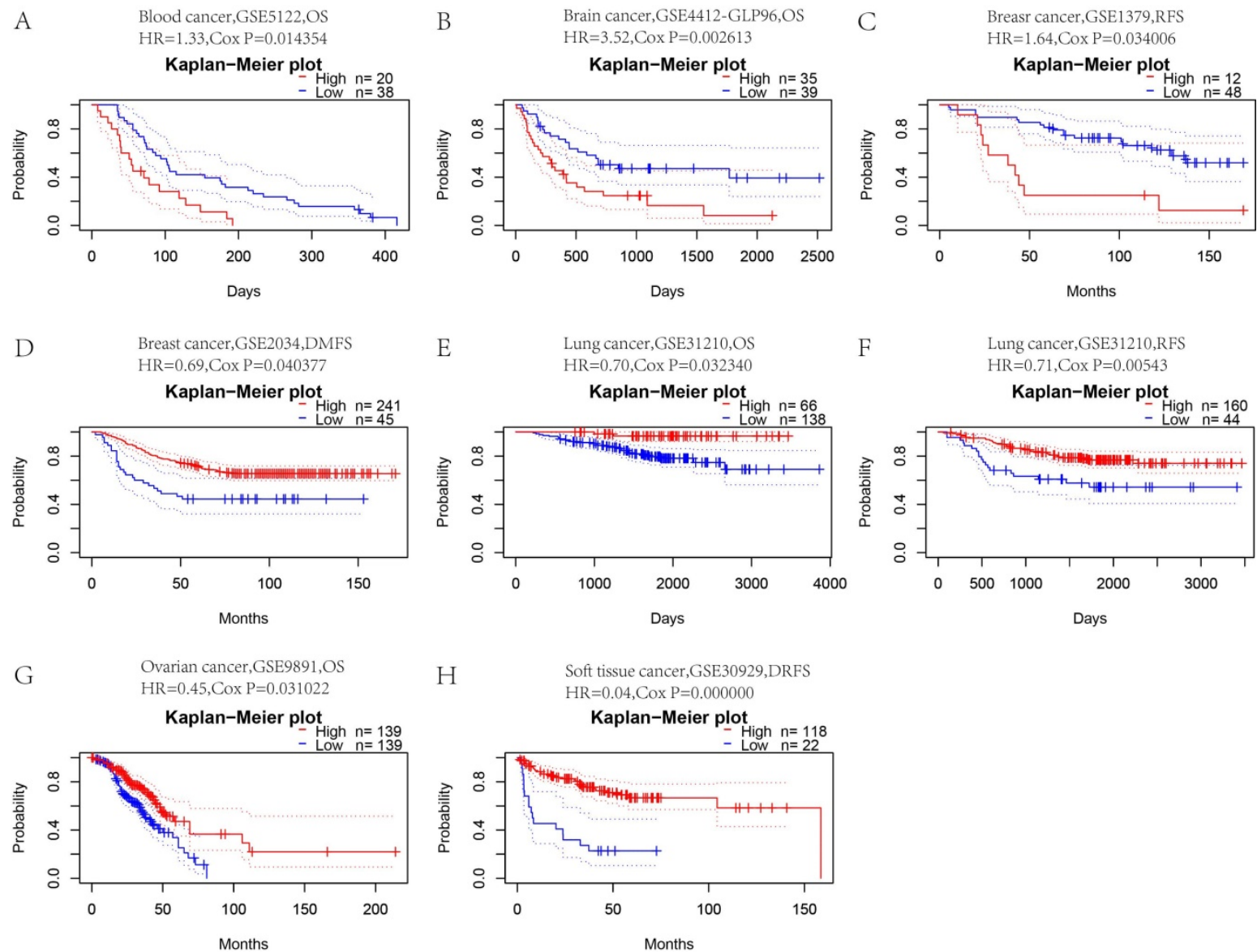

Figure 3. Kaplan-Meier survival curves comparing high and low expression of CFP in different cancers in the PrognoScan. $(A)$ OS ( $n=58)$ in blood cancer cohort GSE5122. (B) OS $(n=74)$ in brain cancer cohort GSE4412-GLP96. (C.D) RFS $(n=60)$ in breast cancer cohort GSE1379 and DMFS ( $n=286)$ in breast cancer cohort GSE2034. $(E, F)$ OS $(n=204)$ and RFS $(n=204)$ in lung cancer cohort GSE31210. $(G)$ OS $(n=278)$ in ovarian cancer cohort GSE9891. $(H)$ DRFS $(n=140)$ in soft tissue cancer cohort GSE30929. DSS, disease-specific survival; OS, overall survival; DMFS, distant metastasis-free survival; DFS, disease-free survival; RFS, relapse-free survival.

\section{Low CFP expression impacts the stratified STAD population}

In order to explore the relationship between CFP and gastric cancer, and its possible mechanism in gastric cancer, we used the Kaplan-Meier plotter database to analyze the relationship between CFP expression and clinical characteristics of gastric cancer patients. Low expression of CFP was associated with worse survival in male and female patients as well as Lauren classification and differentiation $(P<0.05)$. Besides, in stage 3, stage T3, stage N2 and N3, low expression of CFP was associated with worse survival in gastric cancer $(P<0.05)$.

\section{CFP expression is correlated with immune infiltration level in LUAD and STAD}

Infiltration of related immune cells in the tumor microenvironment is an independent predictor of survival and prognosis. Therefore, we analyzed the coefficients of CFP and immune infiltration levels of different tumors in the TIMER to determine how tumor immune infiltration levels are related to the expression of CFP. The results showed that the expression of CFP was correlated with the infiltration level of B cells in 23 tumors, CD8+ T cells in 20 tumors, CD4+ T cells in 31 tumors, macrophages in 20 tumors, neutrophils in 25 tumors, and dendritic cells in 27 tumors respectively (Supplementary Figure 1). Tumor purity is an important factor for the analysis of immune infiltration in clinical tumor samples by genomic approaches. The analysis results showed that CFP expression in 29 Tumor types was significantly correlated with tumor purity. Interestingly, we found that CFP expression level was correlated with poorer prognosis and high immune infiltration in LUAS and STAD. Especially in LUAD, the CFP expression level had significant positive correlations with the infiltration level of $B$ cells $(R=0.354, P=1.04 \mathrm{e}-15)$, CD8+ T cells $(\mathrm{R}=0.18, P=6.72 \mathrm{e}-05), \mathrm{CD} 4+\mathrm{T}$ cells $(\mathrm{R}$ $=0.45, P=1.49 \mathrm{e}-25)$, macrophages $(\mathrm{R}=0.34, P=$ 1.28e-14), neutrophils ( $R=0.417, P=9.91 \mathrm{e}-22)$, and dendritic cells $(\mathrm{R}=0.445, P=3.90 \mathrm{e}-25)$ (Figure 5). CFP expression level had significant positive correlations with the infiltration levels of CD8+ T cells $(\mathrm{R}=0.454, P$ $=3.48 \mathrm{e}-20), \mathrm{CD} 4+\mathrm{T}$ cells $(\mathrm{R}=0.456, P=3.13 \mathrm{e}-20)$, macrophages $(\mathrm{R}=0.459, P=1.22 \mathrm{e}-20)$, neutrophils ( $\mathrm{R}$ $=0.578, P=1.83 \mathrm{e}-34)$, and dendritic cells $(\mathrm{R}=0.613, P$ 
$=1.38 \mathrm{e}-39$ ), but had no significant correlation with $\mathrm{B}$ cell in STAD (Figure 5). These findings strongly suggested that CFP plays a specific role in immune infiltration in LUAD and STAD.

\section{Correlation analysis between CFP expression and immune markers}

To further explore the potential relationships between CFP and infiltrating immune cells, we examined the correlations between $C F P$ and several immune cell markers in TIMER and GEPIA (Supplementary Table 1, Figure 6). These markers were used to characterize immune cells, including B cells, CD8+ $\mathrm{T}$ cells, M1/M2 macrophages, tumor-associated macrophages, monocytes, NK, neutrophils, and DCs. In particular, we analyzed the correlation between CFP expression and T cells with different functions, such as Th1, Th2, Tfh, Th17, Tregs

C STAD,PPS

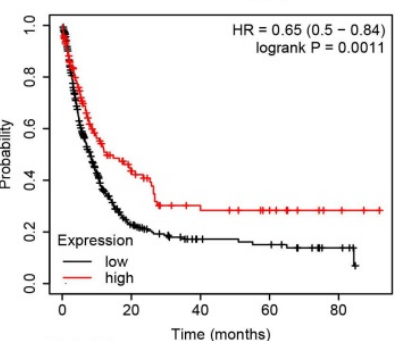

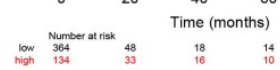

G

F

LUAD,PPS

E $\quad \begin{aligned} & \text { LUAD,FP } \\ & \text { CFP (206380_s_at) }\end{aligned}$
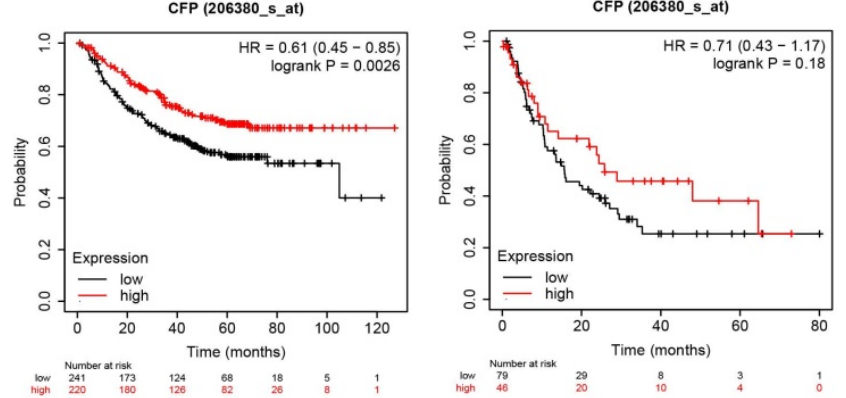

LUSC, OS

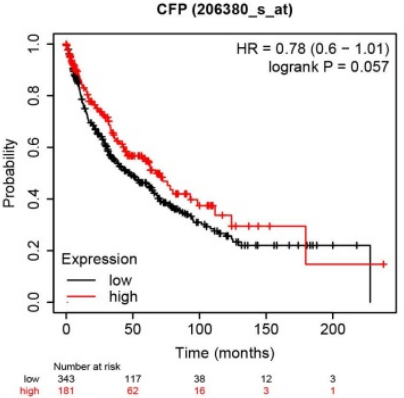

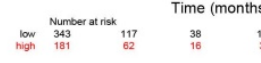

K

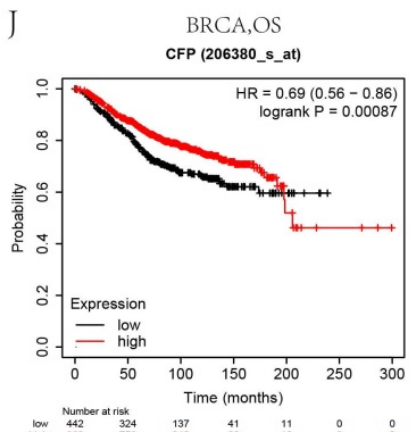

K $\begin{gathered}\text { BRCA,PPS } \\ \text { CFP (206380_s_at) }\end{gathered}$

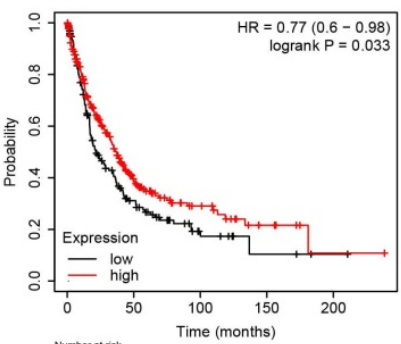

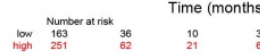

$\mathrm{O}$

N

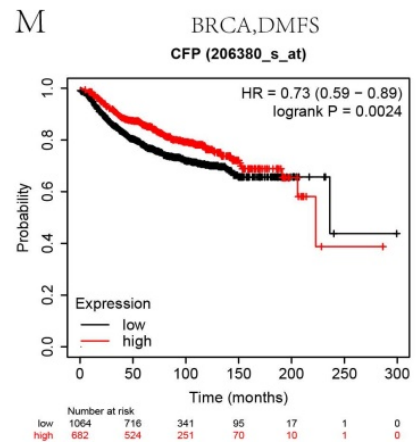

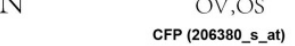

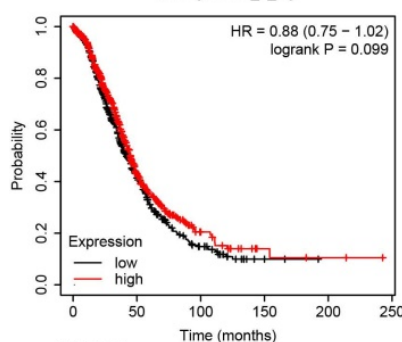

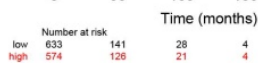

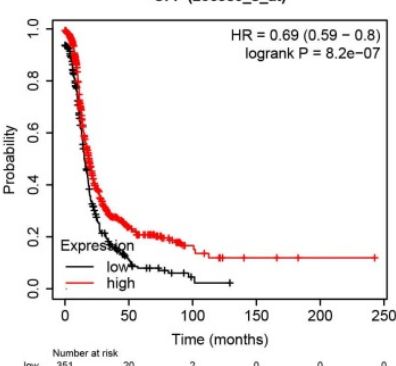

D LUAD,OS

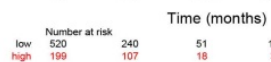

$\mathrm{H}$

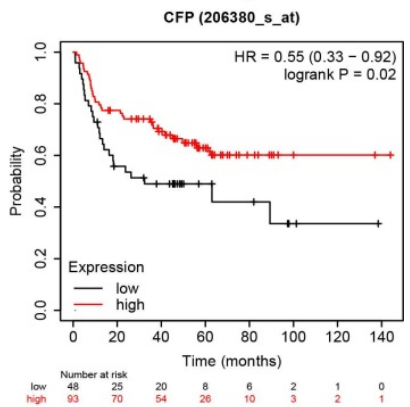

L
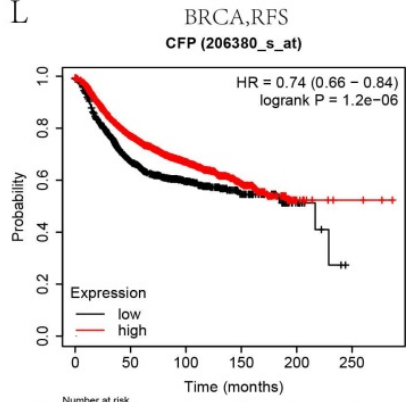

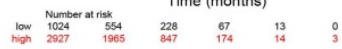

$\mathrm{P}$

P OV,PPS

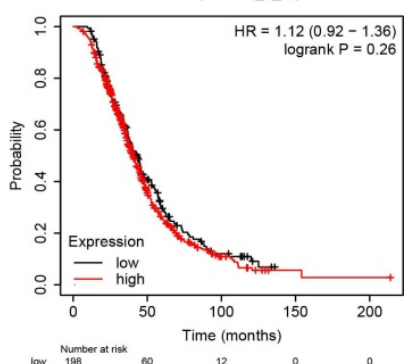

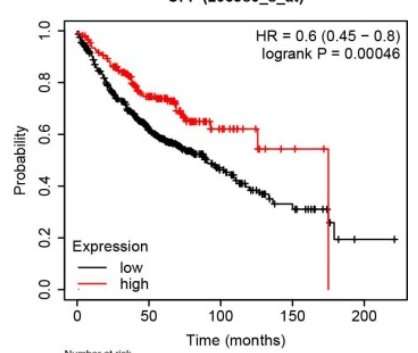

Figure 4. Kaplan-Meier survival curves comparing the high and low expression of CFP in different cancers in Kaplan-Meier Plotter. OS, FP and PPS of STAD (A, B, C), LUAD (D, E, F), LUSC (G, H, I); OS, PPS, RFS and DMFS of BRCA (J, K, L, M); OS, PFS, PPS of OV (N, O, P). Red curve represents patients with high expression of CFP. FP, First progression; OS, overall survival; PPS, Post Progression Survival; DMFS, distant metastasis-free survival; DFS, disease-free survival; RFS, relapse-free survival. 
and failing $\mathrm{T}$ cells. The results showed that CFP expression levels were significantly correlated with most of the immune marker sets of different $T$ cells in STAD and LUAD. Interestingly, we found B cells and macrophages were two immune cell types most strongly correlated with CFP expression in STAD and LUAD. Therefore, we further analyzed the correlations of CFP expression and B cell/macrophage markers in tumor tissues of LUAD and STAD in GEPIA (Table 2). The correlation between CFP and monocytes and TAMs markers is similar to the data analysis results in TIMER, suggesting that CFP may regulate the polarization of macrophages in LUAD and STAD. Besides, in STAD, the correlation between CFP and M2 Macrophage is more significant compared with M1 Macrophage, which is different from LUAD. These might help explain the differences in patient survival (Table 2). We also found significant correlations between CFP and marker genes of Treg and $\mathrm{T}$ cell exhaustion (Supplementary Table 1). These results further confirmed the findings that CFP was specifically correlated with immune infiltrating cells in LUAD and STAD which suggested that CFP plays a vital role in immune escape in LUAD and STAD microenvironment.

\section{Discussion}

Complement Activation is not only an integral part of innate immunity, but also an established defense mechanism against the invasion of pathogens. Meanwhile, complement is involved in the processes of acquired immune response, inflammation, hemostasis, embryogenesis and organ repair and development [13, 14], which is closely related to tumor progression. Related clinical data had shown that complement activation is at least partially involved in the progression of non-small cell lung cancer [15]. Several recent studies also confirmed that complement plays an active role in regulating $\mathrm{T}$ cell immunity $[16,17]$ and natural killer cells are also bound to CFP through NKp46 receptor [18]. CFP is an important positive regulator of alternative pathway (AP) which acts to stabilize C3 and C5 invertase, and is associated with the destruction of bacteria, neutralization of viruses, and cytolysis of certain red blood cells, and as an important component of the complement substitution activation pathway [19]. However, few researches had been done on the relationship between CFP and tumor so far with more studies are focused on non-neoplastic disease. For instance, the high risk of severe Neisseria meningitides infection is associated with new mutations in the CFP gene [20, 21]. Therefore, the potential relationship between CFP and tumor prognosis and immunologic invasion was analyzed in our study.

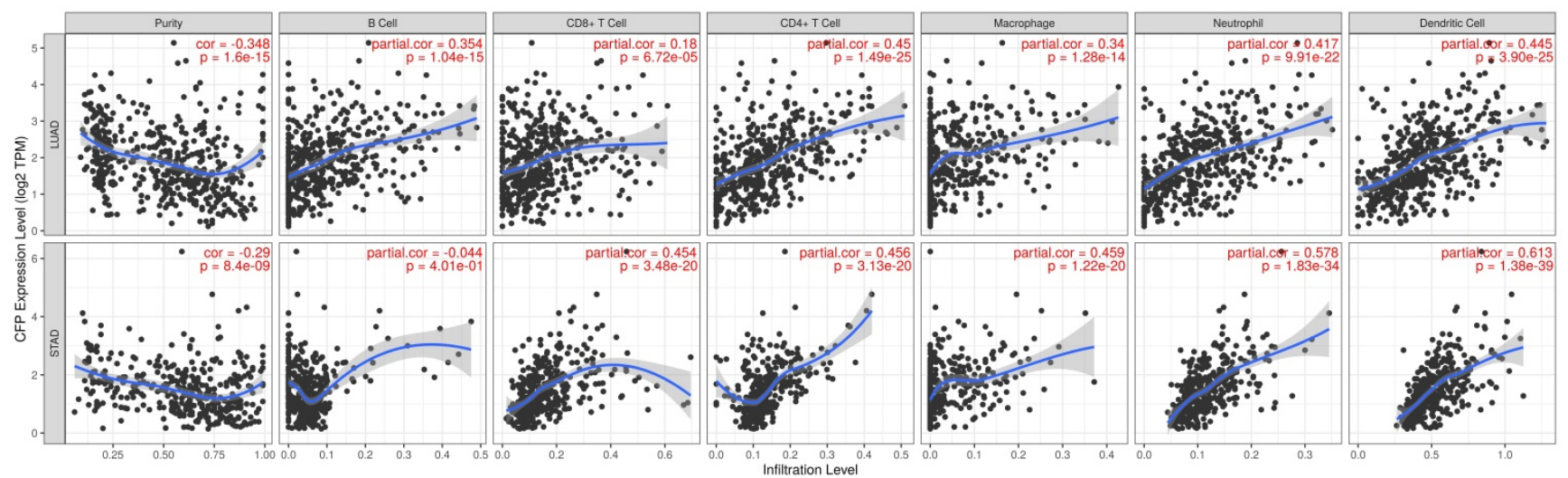

Figure 5. Correlation of CFP expression with immune infiltration level in LUAD and STAD. CFP expression is significantly negatively related to tumor purity and has significant positive correlations with infiltrating levels of B cells, CD8+ T cells, CD4+ T cells, macrophages, neutrophils, and dendritic cells in LUAD. CFP expression is significantly negatively related to tumor purity and has significant positive correlations with infiltrating levels of CD8+ T cells, CD4+ T cells, macrophages, neutrophils, and dendritic cells in STAD, other than B cells.

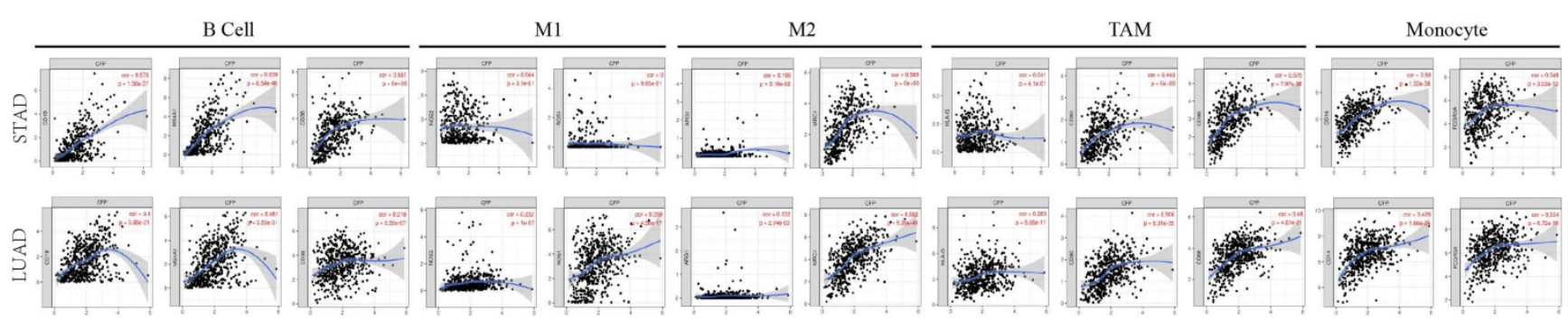

Figure 6. CFP expression correlates with B cell infiltration and macrophage polarization in STAD and LUAD. Relations between CFP expression and immune markers including CD19, MS4A1, and CD38 of B cell, NOS2 and ROS1 of M1 macrophage, ARG1 and MRC1 of M2 macrophage, HLA-G, CD80, and CD86 of TAM, and CD14 and CD16 of monocyte. STAD, stomach adenocarcinoma; LUAD, lung adenocarcinoma; TAM, tumor-associated-macrophages. $P<0.05$ is considered as significant. 
Table 1. Correlation of CFP mRNA expression and clinical prognosis in gastric cancer with different clinicopathological characteristics by Kaplan-Meier plotter

\begin{tabular}{|c|c|c|c|c|c|c|}
\hline \multirow[t]{2}{*}{$\begin{array}{l}\text { Clinicopathological } \\
\text { characteristics }\end{array}$} & \multicolumn{3}{|c|}{$\begin{array}{l}\text { Overall survival } \\
(\mathrm{n}=875)\end{array}$} & \multicolumn{3}{|c|}{$\begin{array}{l}\text { Progression-free survival } \\
(\mathrm{n}=640)\end{array}$} \\
\hline & $\mathrm{N}$ & Hazard ratio & $\begin{array}{l}P \text { - } \\
\text { value }\end{array}$ & $\mathrm{N}$ & Hazard ratio & $\begin{array}{l}P \text { - } \\
\text { value }\end{array}$ \\
\hline \multicolumn{7}{|l|}{ Sex } \\
\hline Female & 236 & $0.65(0.44-0.98)$ & 0.0386 & 201 & $0.61(0.39-0.95)$ & 0.0259 \\
\hline Male & 544 & $0.68(0.54-0.84)$ & 0.0005 & 437 & $0.75(0.59-0.96)$ & 0.0202 \\
\hline \multicolumn{7}{|l|}{ Stage } \\
\hline 1 & 67 & $0.42(0.16-1.113)$ & 0.0762 & 60 & $0.53(0.18-1.58)$ & 0.2474 \\
\hline 2 & 140 & $2(0.99-4.05)$ & 0.0501 & 131 & $1.87(0.94-3.72)$ & 0.0686 \\
\hline 3 & 305 & $0.45(0.31-0.64)$ & $6.50 \mathrm{E}-06$ & 186 & $0.54(0.35-0.83)$ & 0.0048 \\
\hline 4 & 148 & $1.37(0.93-2.01)$ & 0.1056 & 141 & $1.27(9.87-1.86)$ & 0.2194 \\
\hline \multicolumn{7}{|l|}{ Stage $T$} \\
\hline 2 & 241 & $1.45(0.9-2.33)$ & 0.1293 & 239 & $1.55(0.97-2.47)$ & 0.064 \\
\hline 3 & 204 & $0.6(0.41-0.88)$ & 0.0087 & 204 & $0.62(0.43-0.9)$ & 0.0101 \\
\hline 4 & 38 & $0.42(0.18-1.02)$ & 0.0478 & 39 & $0.5(0.23-1.08)$ & 0.0708 \\
\hline \multicolumn{7}{|l|}{ Stage $\mathbf{N}$} \\
\hline 0 & 74 & $0.68(0.28-1.65)$ & 0.3911 & 72 & $0.71(0.29-1.74)$ & 0.4548 \\
\hline 1 & 225 & $1.46(0.94-2.26)$ & 0.0879 & 222 & $1.41(0.93-2.13)$ & 0.106 \\
\hline 2 & 121 & $0.3(0.17-0.54)$ & $1.70 \mathrm{E}-05$ & 125 & $0.42(0.25-0.7)$ & 0.0007 \\
\hline 3 & 76 & $2.04(1.14-3.65)$ & 0.0138 & 76 & $1.69(0.99-2.88)$ & 0.0508 \\
\hline $1+2+3$ & 422 & $0.78(0.57-1.05)$ & 0.1004 & 423 & $0.81(0.61-1.08)$ & 0.1517 \\
\hline \multicolumn{7}{|l|}{ Stage M } \\
\hline 0 & 444 & $0.78(0.58-1.07)$ & 0.1194 & 443 & $1.22(0.89-1.68)$ & 0.2086 \\
\hline 1 & 56 & $1.49(0.83-2.66)$ & 0.1781 & 56 & $0.62(0.33-1.15)$ & 0.1252 \\
\hline \multicolumn{7}{|c|}{ Laurgen Classification } \\
\hline Intestinal & 320 & $1.28(0.9-1.82)$ & 0.1697 & 263 & $1.34(0.91-1.99)$ & 0.1358 \\
\hline Diffuse & 241 & $0.53(0.35-0.79)$ & 0.0017 & 231 & $0.48(0.31-0.73)$ & 0.0005 \\
\hline \multicolumn{7}{|l|}{ Differentiation } \\
\hline Poor & 165 & $0.53(0.34-0.81)$ & 0.0032 & 121 & $0.48(0.29-0.78)$ & 0.0027 \\
\hline Moderate & 67 & $1.31(0.61-2.81)$ & 0.4959 & 67 & $1.52(0.71-3.24)$ & 0.2736 \\
\hline
\end{tabular}

Table 2. Correlations between CFP and genes markers of B cells, macrophages, and monocytes in GEPIA

\begin{tabular}{|c|c|c|c|c|c|c|c|c|c|}
\hline \multirow[t]{3}{*}{ Celltype } & \multirow{3}{*}{$\begin{array}{l}\text { Gene } \\
\text { marker }\end{array}$} & \multicolumn{4}{|c|}{ LUAD } & \multicolumn{4}{|c|}{ STAD } \\
\hline & & \multicolumn{2}{|c|}{ Tumor } & \multicolumn{2}{|c|}{ Normal } & \multicolumn{2}{|c|}{ Tumor } & \multicolumn{2}{|c|}{ Normal } \\
\hline & & $\overline{\mathrm{R}}$ & $P$ & $\mathrm{R}$ & $P$ & $\mathrm{R}$ & $P$ & $\mathrm{R}$ & $P$ \\
\hline \multirow[t]{3}{*}{ B cell } & CD19 & 0.21 & $* * *$ & 0.11 & 0.4 & 0.29 & $* * *$ & 0.62 & $* * *$ \\
\hline & $\mathrm{CD} 20$ & 0.23 & $* * *$ & 0.066 & 0.62 & 0.31 & $* * *$ & 0.68 & $* * *$ \\
\hline & CD38 & 0.0081 & 0.86 & -0.092 & 0.49 & 0.081 & 0.1 & 0.48 & $* *$ \\
\hline \multirow[t]{2}{*}{ M1 } & NOS2 & -0.014 & 0.76 & 0.2 & 0.13 & -0.066 & 0.18 & 0.051 & 0.77 \\
\hline & ROS & 0.16 & $* * *$ & -0.2 & 0.14 & -0.039 & 0.44 & 0.17 & 0.32 \\
\hline \multirow[t]{2}{*}{ M2 } & ARG1 & 0.022 & 0.63 & 0.29 & * & 0.1 & * & -0.37 & * \\
\hline & MRC1 & 0.43 & $* * *$ & 0.24 & 0.064 & 0.2 & $* * *$ & 0.34 & * \\
\hline \multirow[t]{3}{*}{ TAM } & HLA-G & 0.009 & 0.84 & -0.09 & 0.5 & -0.017 & 0.73 & -0.1 & 0.55 \\
\hline & CD80 & 0.28 & $* * *$ & 0.097 & 0.47 & 0.14 & $* *$ & 0.64 & $* * *$ \\
\hline & CD86 & 0.34 & $* * *$ & 0.29 & * & 0.24 & $* * *$ & 0.72 & $* * *$ \\
\hline \multirow[t]{2}{*}{ Monocyte } & CD14 & 0.33 & $* * *$ & 0.54 & $* * *$ & 0.32 & $* * *$ & 0.7 & $* * *$ \\
\hline & CD16 & 0.2 & $* * *$ & 0.075 & 0.57 & 0.048 & 0.33 & 0.5 & $* *$ \\
\hline
\end{tabular}

STAD, stomach adenocarcinoma; LUAD, lung adenocarcinoma. TAM,

tumor-associated-macrophage; Tumor, correlation analysis in tumor tissue of TCGA; Normal, correlation analysis in normal tissue of TCGA. ${ }^{*} P<0.01$; ** $P<$ $0.001 ; * * * P<0.0001$

In this study, we used TCGA data from Oncomine and TIMER database to explore the expression of CFP in different tumors. Comparing with normal tissues, CFP is highly expressed in kidney cancer, leukemia, and lymphoma, while being significantly decreased in bladder cancer, brain cancer, and central nervous system cancer, breast cancer, colorectal cancer, leukemia, liver cancer, lung cancer, myeloma, ovarian cancer, and sarcoma (Figure 1A). Meanwhile, relatively low expression of CFP was observed in BLCA, BRCA, CHOL, COAD, HNSC, KICH, LHIC, and LUAD, LUSC, READ, STAD while high expression was observed in HNSC-HPV-positive and KIRC (Figure 1B). In order to detect the protein expression level of CFP, we used the UALCAN Database and found that the protein expression level of CFP is low in BRCA, COAD, LUAD, OV and UCEC (Figure 2A-F). Besides, immunohistochemical staining results confirmed that the protein expression levels of CFP were significantly decreased in LUAD and STAD compared with the normal tissues (Figure 2G, H). The difference in expression of CFP in different tumors may be the result of difference in data collection methods, or more likely, suggest that different tumors have different biological properties. Studies on transgenic mice have shown that CFP can be used as a general serum biomarker for lung cancer [22]. In addition, a study was also conducted on 21 benzamidine operators who were tested for serum properdin levels over time and found that serum properdin level was negatively correlated with the risk of bladder cancer, which is consistent with the results of our analysis, suggesting that CFP may serve as a prognostic biomarker [23].On this basis, we explored the relationship between $C F P$ and tumor prognosis using independent and tumor prognosis-related data and found that the expression of CFP in LUAD and STAD had the same prognostic value. In six groups of prognostic data, CFP expression level was negatively correlated with breast, lung, ovarian and soft tissue cancer prognosis, suggesting that low CFP expression can serve as an independent risk factor for the above tumors (Figure 3). Kaplan- Meier mapper data analysis showed that low expression of CFP was also associated with poor prognosis for STAD, LUAD, and BRCA (Figure 4). In particular, of the different stages of gastric cancer, low expression of CFP was associated with poor prognosis at stage 3, T3, N2 and N3 (Table 1). Interestingly, only small-scale invasion occurred in all of these stages as no invasion occurred in adjacent structures and no distant metastasis was observed, suggesting that low expression of CFP may be only associated with the initial stages of metastasis, and no significant correlation between CFP and survival rate was observed after extensive cancer metastasis (Table 1). In addition, low expression of CFP was also significantly associated with poor prognosis in severe low-grade and diffuse-type gastric cancer. These findings strongly suggest that CFP can be used as an independent marker for prognosis in gastric cancer. 
Another major finding of this study was that CFP expression was associated with different levels of immune infiltration of tumors, STAD and LUAD in particular. Tumor infiltrating lymphocytes (TIL) include $\mathrm{T}$ cells, $\mathrm{B}$ cells and NK cells. In STAD, especially among advanced patients, the percentage of these cells in TIL was significantly increased, suggesting that they may be related to tumor immune escape phenomena and that TIL shows $\mathrm{T}$ cell dysfunction in STAD [24]. The results of our analysis showed that, the CFP expression in LUAD and STAD was positively correlated with the number of CD8+ T cells, CD4+ T cells, macrophages, neutrophils and dendritic cells and negatively correlated with immune infiltration (Figure 5), suggesting that the CFP may be involved in the immunomodulatory mechanisms of LUAD and STAD. Macrophages are the most typical tumor-infiltrating immune cells that play an important role in the tumor microenvironment. In the last decade, numerous studies have shown that the auto infiltration or polarization pathway of tumorassociated macrophages (TAMs) has the potential to become the new targets of malignant tumor treatment [25].The results of this study indicate that CFP expression is closely correlated to TAMs while being less correlated to M1 comparing M2 (Supplementary Table 1, Table 2) and M2 phenotype of TAMs generally considered more resemble primary tumors in terms of function [26]. Early studies have shown that macrophage polarization is more skewed toward M2 in the absence of CFP [27-29]. These results suggested that there may be a regulatory role of $C F P$ on the polarization of TAM, especially towards M2. The current study showed that a variety of $T$ cells play a role in TME. Particularly, CD8+ T cells and Th1 cells play the role of antitumor immune effector cells in several types of solid tumors and are associated with favorable prognosis [30]. Besides, our results indicate that there is a moderate to strong positive relationships between CFP expression level and infiltration level of $\mathrm{CD} 8+\mathrm{T}, \mathrm{CD} 4+\mathrm{T}$ cell and neutrophils in STAD and LUAD (Figure 5). Moreover, these results reveal the potential regulating role of CFP in $\mathrm{T}$ cell and neutrophils, which including the increase of CFP expression positively correlates with the expression of neutrophils and T cell (general) gene markers (MPO, CD15, CD66B, CD11b, CCR7, CD3D, CD3E, CD2) in STAD and LUAD. Neutrophils play an important role in both chemically mediating inflammatory response through myeloperoxidase (MPO) and biologically promoting metastasis during inflammation triggered by the primary tumor or environmental stimuli [31]. CD11b is implicated in various adhesive interactions of monocytes, macrophages and granulocytes as well as in regulating neutrophil migration $[32,33]$. CD3D, CD3E are the part of T-cell receptor (TCR)-CD3 complex present on T-lymphocyte cell surface, play an essential role in signal transduction in T cell activation and in thymocyte differentiation [34, 35]. CD2 interacts with lymphocyte function-associated antigen CD58 (LFA-3) and CD48/BCM1 to mediate adhesion between T-cells and other cell types. Downregulation of CD2 may attenuate the antitumor T cell response, which has implications for checkpoint immunotherapy [36]. Additionally, This study also provided evidence that CFP expression level in cancers are significantly correlated with APCs, APCs can assist and modulate $\mathrm{T}$ cell function, speculating whether CFP indirectly affects $\mathrm{T}$ cell function through APCs. Therefore, it is reasonable to speculate that CFP-mediated changes in the function of immune cells, in particular, the functions of T-lymphocyte activation and neutrophil adhesion, may be closely related to immune infiltration, thereby affecting the tumor microenvironment. The effects of tumor-infiltrating immune cells have been debated for decades, and to our knowledge, these immune cells, according to the complex signals of TME, play a dual role, potentially inhibiting, as well as promoting, malignant tumor development, though more details are subject to further research $[37,38]$. Our results suggested that CFP may also play an important role in the recruitment and regulation of immune infiltrating cells in gastric and lung cancers.

Promotion of complement activity by properdin results in changes on the tumor microenvironment that contribute to innate and adaptive immune responses, including immune cell infiltration, antigen presenting cell maturation, pro-inflammatory cytokine production, and tissue damage [19]. Neutrophils store and rapidly release their intracellular properdin into the extracellular space, in response to plenty of inflammatory agonists including the cytokines TNF- $\alpha$, IL-8 (CXCL8), IFN- $\alpha$, bacterial LPS, C5a, etc. [39, 40]. Since alternative pathway (AP) accounts for about $80-90 \%$ of the terminal pathway activity initiated by classical pathways $(\mathrm{CP})$ and lectin pathways (LP), inhibition of properdin may effectively limit inflammation-mediated injury in diseases in which CP and LP play pathogenic roles [19]. There are many experimental evidences support the possibility that inhibiting properdin may be a promising approach for the treatment of inflammatory diseases [41, 42]. Inflammation is a recognized marker of cancer and contributes to the development and progression of malignant tumors to a large extent [43]. However, properdin inhibition significantly increase susceptibility to Neisseria meningitidis and septicemia, as well as increasing the 
risk that converts C3 glomerulopathy to a lethal, rapidly developing C3 glomerulopathy [20, 44]. In terms of cancer, this raises the possibility that the supplementation of properdin may be beneficial in treatment. To support this notion, there are many researches indicate that transfected HEK293 cell expressing membrane-bound properdin [45], properdin-coated nanoparticles initiate complement activation [46], and properdin insufficiency promotes a microenvironment that helps tumor cells evade the immune response [27]. Compared with these studies, our current results indicate that CFP may play a crucial role in the recruitment and regulation of cancer immune infiltrating cells, ultimately affecting the patient prognosis. Therefore, future studies are needed to be focus on the mechanism of CFP, at both cellular and molecular levels, will be helpful to clarify the role of CFP in inflammatory and treatment of cancers.

Recent studies have provided a possible mechanism to explain why CFP is associated with poor prognosis and immune infiltration of tumors. On the one hand, CFP may exercise direct intervention on tumor microenvironment. Previous studies have shown that CFP is a ligand of NK cell activation receptor NKp46 [18], indicating that CFP can not only remove tumor cells through complement activation, but also promote the removal of tumor cells by promoting phagocytosis and removal of target cells without activated complement. This may work in the same mechanism as the role of CFP on TAM polarization as found in our analysis results. In addition, CFP can recognize dead $\mathrm{T}$ cells by specific surface proteoglycan and mediate their opsonization and phagocytosis while it plays similar role in the process of apoptosis of other cell types [47]. To demonstrate this point, a recent study showed that CFP can inhibit the growth of cancer cells in breast cancer through TES mediated up-regulation of DDIT3 [12]. On the other hand, CFP may affect the tumor microenvironment through the alternative pathway of complement system [13].

Although we have some interesting conclusions, this study still had limitations. First, it completely relies on the open-access database for data acquisition, there will inevitably be systematic bias between the databases, and more accurate experiments such as single cell RNA sequencing are needed for verification. Second, even though the expression of $C F P$ was found to be related to immune cell infiltration and the patient survival in tumor, this study could not prove that CFP affected patient survival through immune infiltration, which needs to be verified by in vivo and in vitro experiments. Third, the evaluation of CFP expression was based on the
mRNA level in the above multiple databases, might not reflect the level of functional protein. Despite we used the UALCAN database to prove that CFP mRNA and protein expression were uniformly low in LUAD, BRCA, COAD and OV, the relationship between mRNA and protein level in other cancers including STAD could not be proved.

\section{Conclusion}

In summary, the low expression of CFP is closely related to the prognosis of multiple tumors (especially lung cancer and gastric cancer), and is associated with increased infiltration of immune cells such as CD8+ T cells, CD4+ T cells, macrophages and neutrophils. In tumor microenvironment, CFP may be involved in the regulation of tumor-related macrophages (TAMs), dendritic cells (DCs), $\mathrm{T}$ cell failure and Tregs. Therefore, CFP may be expected to be an independent risk factor for the prognosis of lung cancer and gastric cancer, and may be involved in the regulation of relevant immune mechanisms in the tumor microenvironment, which need to be further identified in more clinical trials and basic experiments.

\section{Supplementary Material}

Supplementary figures and tables. http://www.jcancer.org/v12p3378s1.pdf

\section{Acknowledgements}

\section{Funding}

This work was supported by Project of Administration of Traditional Chinese Medicine of Jiangsu Province of China (YB2015166).

\section{Data availability statement}

We confirm that my article contains a Data Availability Statement. We confirm that we have included a citation for available data in my references section. All the data in this study are available from Oncomine database (https://www.oncomine.org), TIMER database (https://cistrome.shinyapps.io/ timer/), PrognoScan database (http://www.abren. net/PrognoScan/), Kaplan-Meier plotter database (http://kmplot.com/analysis/) and GEPIA database (http://gepia.cancer-pku.cn/).

\section{Competing Interests}

The authors have declared that no competing interest exists.

\section{References}

1. Zheng RS, Sun KX, Zhang SW, Zeng HM, Zou XN, Chen R, Gu XY, Wei WW, He J: [Report of cancer epidemiology in China, 2015]. Zhonghua zhong liu za zhi [Chinese journal of oncology] 2019, 41(1):19-28. 
2. Bray F, Ferlay J, Soerjomataram I, Siegel RL, Torre LA, Jemal A: Global cancer statistics 2018: GLOBOCAN estimates of incidence and mortality worldwide for 36 cancers in 185 countries. CA: a cancer journal for clinicians 2018, 68(6):394-424.

3. Chen W, Zheng R, Baade PD, Zhang S, Zeng H, Bray F, Jemal A, Yu XQ He J: Cancer statistics in China, 2015. CA: a cancer journal for clinicians 2016, 66(2):115-132.

4. Altorki NK, Markowitz GJ, Gao D, Port JL, Saxena A, Stiles B, McGraw $\mathrm{T}$, Mittal V: The lung microenvironment: an important regulator of tumour growth and metastasis. Nature reviews Cancer 2019, 19(1):9-31.

5. Huang T, Song C, Zheng L, Xia L, Li Y, Zhou Y: The roles of extracellular vesicles in gastric cancer development, microenvironment, anti-cancer drug resistance, and therapy. Molecular cancer 2019, 18(1):62.

6. Hinshaw DC, Shevde LA: The Tumor Microenvironment Innately Modulates Cancer Progression. Cancer research 2019, 79(18):4557-4566.

7. Pitt JM, Vétizou M, Daillère R, Roberti MP, Yamazaki T, Routy B, Lepage P, Boneca IG, Chamaillard M, Kroemer G et al: Resistance Mechanisms to Immune-Checkpoint Blockade in Cancer: Tumor-Intrinsic and -Extrinsic Factors. Immunity 2016, 44(6):1255-1269.

8. Michels M, Volokhina EB, van de Kar N, van den Heuvel L: The role of properdin in complement-mediated renal diseases: a new player in complement-inhibiting therapy? Pediatric nephrology (Berlin, Germany) 2019, 34(8):1349-1367.

9. Gordon SR, Maute RL, Dulken BW, Hutter G, George BM, McCracken MN, Gupta R, Tsai JM, Sinha R, Corey D et al: PD-1 expression by tumour-associated macrophages inhibits phagocytosis and tumour immunity. Nature 2017, 545(7655):495-499.

10. Muro K, Chung HC, Shankaran V, Geva R, Catenacci D, Gupta S, Eder JP, Golan T, Le DT, Burtness B et al: Pembrolizumab for patients with PD-L1-positive advanced gastric cancer (KEYNOTE-012): a multicentre, open-label, phase $1 \mathrm{~b}$ trial. The Lancet Oncology 2016, 17(6):717-726.

11. Topalian SL, Drake CG, Pardoll DM: Immune checkpoint blockade: a common denominator approach to cancer therapy. Cancer cell 2015, 27(4):450-461.

12. Block I, Müller C, Sdogati D, Pedersen H, List M, Jaskot AM, Syse SD, Lund Hansen P, Schmidt S, Christiansen H et al: CFP suppresses breast cancer cell growth by TES-mediated upregulation of the transcription factor DDIT3. Oncogene 2019, 38(23):4560-4573.

13. Afshar-Kharghan V: The role of the complement system in cancer. The Journal of clinical investigation 2017, 127(3):780-789.

14. Kwak JW, Laskowski J, Li HY, McSharry MV, Sippel TR, Bullock BL, Johnson AM, Poczobutt JM, Neuwelt AJ, Malkoski SP et al: Complement Activation via a C3a Receptor Pathway Alters CD4(+) T Lymphocytes and Mediates Lung Cancer Progression. Cancer research 2018, 78(1):143-156

15. Kleczko EK, Kwak JW, Schenk EL, Nemenoff RA: Targeting the Complement Pathway as a Therapeutic Strategy in Lung Cancer. Frontiers in immunology 2019, 10:954.

16. Girardi G, Lingo JJ, Fleming SD, Regal JF: Essential Role of Complement in Pregnancy: From Implantation to Parturition and Beyond. Frontiers in immunology 2020, 11:1681.

17. West EE, Kemper C: Complement and T Cell Metabolism: Food for Thought. Immunometabolism 2019, 1(T Cell Metabolic Reprogramming):e190006.

18. Narni-Mancinelli E, Gauthier L, Baratin M, Guia S, Fenis A, Deghmane AE, Rossi B, Fourquet P, Escalière B, Kerdiles YM et al: Complement factor $\mathrm{P}$ is a ligand for the natural killer cell-activating receptor $\mathrm{NKp} 46$. Science immunology 2017, 2(10):eaam9628.

19. Chen JY, Cortes C, Ferreira VP: Properdin: A multifaceted molecule involved in inflammation and diseases. Molecular immunology 2018, 102:58-72.

20. Ali YM, Hayat A, Saeed BM, Haleem KS, Alshamrani S, Kenawy HI, Ferreira VP, Saggu G, Buchberger A, Lachmann PJ et al: Low-dose recombinant properdin provides substantial protection against Streptococcus pneumoniae and Neisseria meningitidis infection. Proceedings of the National Academy of Sciences of the United States of America 2014, 111(14):5301-5306.

21. Gillet B, Joram N, Bacchi VF, Thomas C, Béné MC, Wuillème S: Neisseria meningitidis inside neutrophils, revealing properdin deficiency. International journal of infectious diseases : IJID : official publication of the International Society for Infectious Diseases 2020, 99:117-118.

22. Chatterji B, Borlak J: A 2-DE MALDI-TOF study to identify disease regulated serum proteins in lung cancer of c-myc transgenic mice. Proteomics 2009, 9(4):1044-1056.

23. Horton AW, Bingham EL: Risk of bladder tumors among benzidine workers and their serum properdin levels. Journal of the National Cancer Institute 1977, 58(5):1225-1228.

24. Kim JY, Kim WG, Kwon CH, Park DY: Differences in immune contextures among different molecular subtypes of gastric cancer and their prognostic impact. Gastric cancer : official journal of the International Gastric Cancer Association and the Japanese Gastric Cancer Association 2019, 22(6):1164-1175.

25. Komohara Y, Fujiwara Y, Ohnishi K, Takeya M: Tumor-associated macrophages: Potential therapeutic targets for anti-cancer therapy. Advanced drug delivery reviews 2016, 99(Pt B):180-185.

26. Rojas A, Araya P, Gonzalez I, Morales E: Gastric Tumor Microenvironment. Advances in experimental medicine and biology 2020, 1226:23-35

27. Al-Rayahi IA, Browning MJ, Stover C: Tumour cell conditioned medium reveals greater M2 skewing of macrophages in the absence of properdin. Immunity, inflammation and disease 2017, 5(1):68-77.

28. Dupont A, Mohamed F, Salehen N, Glenn S, Francescut L, Adib R, Byrne S, Brewin H, Elliott I, Richards L et al: Septicaemia models using Streptococcus pneumoniae and Listeria monocytogenes: understanding the role of complement properdin. Medical microbiology and immunology 2014, 203(4):257-271.

29. Steiner T, Francescut L, Byrne S, Hughes T, Jayanthi A, Guschina I, Harwood J, Cianflone K, Stover C, Francis S: Protective role for properdin in progression of experimental murine atherosclerosis. PloS one 2014, 9(3):e92404.

30. Fridman WH, Pagès F, Sautès-Fridman C, Galon J: The immune contexture in human tumours: impact on clinical outcome. Nature reviews Cancer 2012, 12(4):298-306.

31. Tang L, Wang Z, Mu Q, Yu Z, Jacobson O, Li L, Yang W, Huang C, Kang F, Fan W et al: Targeting Neutrophils for Enhanced Cancer Theranostics. Advanced materials (Deerfield Beach, Fla) 2020, 32(33):e2002739.

32. Losse J, Zipfel PF, Józsi M: Factor $\mathrm{H}$ and factor H-related protein 1 bind to human neutrophils via complement receptor 3 , mediate attachment to Candida albicans, and enhance neutrophil antimicrobial activity. Journal of immunology (Baltimore, Md : 1950) 2010, 184(2):912-921.

33. Bai M, Grieshaber-Bouyer R, Wang J, Schmider AB, Wilson ZS, Zeng L, Halyabar O, Godin MD, Nguyen HN, Levescot A et al: CD177 modulates human neutrophil migration through activation-mediated integrin and chemoreceptor regulation. Blood 2017, 130(19):2092-2100.

34. Martin-Blanco N, Jiménez Teja D, Bretones G, Borroto A, Caraballo M, Screpanti I, León J, Alarcón B, Canelles M: CD3e recruits Numb to promote TCR degradation. International immunology 2016, 28(3):127-137

35. Ngoenkam J, Schamel WW, Pongcharoen S: Selected signalling proteins recruited to the T-cell receptor-CD3 complex. Immunology 2018, 153(1):42-50.

36. Demetriou P, Abu-Shah E, Valvo S, McCuaig S, Mayya V, Kvalvaag A, Starkey T, Korobchevskaya K, Lee LYW, Friedrich M et al: A dynamic CD2-rich compartment at the outer edge of the immunological synapse boosts and integrates signals. Nature immunology 2020, 21(10):1232-1243

37. Binnewies M, Roberts EW, Kersten K, Chan V, Fearon DF, Merad M, Coussens LM, Gabrilovich DI, Ostrand-Rosenberg S, Hedrick CC et al: Understanding the tumor immune microenvironment (TIME) for effective therapy. Nature medicine 2018, 24(5):541-550.

38. Zadka Ł, Grybowski DJ, Dzięgiel P: Modeling of the immune response in the pathogenesis of solid tumors and its prognostic significance. Cellular oncology (Dordrecht) 2020, 43(4):539-575.

39. Camous L, Roumenina L, Bigot S, Brachemi S, Frémeaux-Bacchi V, Lesavre P, Halbwachs-Mecarelli L: Complement alternative pathway acts as a positive feedback amplification of neutrophil activation. Blood 2011, 117(4):1340-1349

40. Uchiyama S, Keller N, Schlaepfer E, Grube C, Schuepbach RA, Speck RF, Zinkernagel AS: Interferon a-Enhanced Clearance of Group A Streptococcus Despite Neutropenia. The Journal of infectious diseases 2016, 214(2):321-328

41. Miwa T, Sato S, Gullipalli D, Nangaku M, Song WC: Blocking properdin, the alternative pathway, and anaphylatoxin receptors ameliorates renal ischemia-reperfusion injury in decay-accelerating factor and CD59 double-knockout mice. Journal of immunology (Baltimore, Md : 1950) 2013, 190(7):3552-3559.

42. Wang Y, Miwa T, Ducka-Kokalari B, Redai IG, Sato S, Gullipalli D, Zangrilli JG, Haczku A, Song WC: Properdin Contributes to Allergic Airway Inflammation through Local C3a Generation. Journal of immunology (Baltimore, Md : 1950) 2015, 195(3):1171-1181.

43. Diakos CI, Charles KA, McMillan DC, Clarke SJ: Cancer-related inflammation and treatment effectiveness. The Lancet Oncology 2014, 15(11):e493-503.

44. Lesher AM, Zhou L, Kimura Y, Sato S, Gullipalli D, Herbert AP, Barlow $\mathrm{PN}$, Eberhardt HU, Skerka C, Zipfel PF et al: Combination of factor $\mathrm{H}$ mutation and properdin deficiency causes severe $\mathrm{C} 3$ glomerulonephritis. Journal of the American Society of Nephrology : JASN 2013, 24(1):53-65. 
45. Vuagnat BB, Mach J, Le Doussal JM: Activation of the alternative pathway of human complement by autologous cells expressing transmembrane recombinant properdin. Molecular immunology 2000, 37(8):467-478.

46. Kouser L, Paudyal B, Kaur A, Stenbeck G, Jones LA, Abozaid SM, Stover CM, Flahaut E, Sim RB, Kishore U: Human Properdin Opsonizes Nanoparticles and Triggers a Potent Pro-inflammatory Response by Macrophages without Involving Complement Activation. Frontiers in immunology 2018, 9:131.

47. Pedersen DV, Rösner T, Hansen AG, Andersen KR, Thiel S, Andersen GR, Valerius T, Laursen NS: Recruitment of properdin by bi-specific nanobodies activates the alternative pathway of complement. Molecular immunology 2020, 124:200-210. 